Revue internationale P.M.E.

Économie et gestion de la petite et moyenne entreprise

\title{
Financement des entreprises et évolution du système financier
}

\section{Henri Jullien et Bernard Paranque}

Volume 8, numéro 3-4, 1995

URI : https://id.erudit.org/iderudit/1008358ar

DOI : https://doi.org/10.7202/1008358ar

Aller au sommaire du numéro

Éditeur(s)

Presses de l'Université du Québec

ISSN

0776-5436 (imprimé)

1918-9699 (numérique)

Découvrir la revue

Citer cet article

Jullien, H. \& Paranque, B. (1995). Financement des entreprises et évolution du système financier. Revue internationale P.M.E., 8(3-4), 43-66.

https://doi.org/10.7202/1008358ar

\section{Résumé de l'article}

L'insuffisante capitalisation souvent évoquée à propos des PMI2 est en grande partie le résultat d'un effort d'investissement relatif similaire, si ce n'est plus fort, que celui de leurs concurrentes de plus grande taille. Elle est imputable, par ailleurs, à la difficulté d'accès aux marchés financiers pour cette catégorie d'entreprises. Pourtant le gain potentiel escompté d'une participation au capital d'une PMI paraît aussi élevé que celui obtenu dans une entreprise de taille plus grande.

Alors que la période 1985-1990 avait permis de développer toutes les techniques de financement sans contraintes particulières, les années récentes ont été marquées par divers facteurs conduisant les banques et, en règle générale, tous les opérateurs financiers à revenir largement sur des pratiques libérales qui avaient facilité l'accès des entreprises aux marchés des capitaux. Face à la montée des risques et à la dégradation des résultats, les banques se sont montrées particulièrement sélectives vis-à-vis des PME. Soit elles ont largement supprimé les financements de secteurs considérés comme sinistrés (immobilier, BTP, informatique...), soit elles n’accordent des crédits qu'avec prudence.

Si l'analyse statistique fait ressortir des situations grandes entreprises/PMI relativement comparables, le constat pour les années récentes montre dans la réalité un niveau de risque sensiblement supérieur.

Par conséquent, au-delà d'un niveau raisonnable de fonds propres que le marché doit pouvoir fournir, la restauration d’un véritable partenariat basé sur la confiance entre banques et entreprises et le partage des risques dans le cadre d'un groupement financier sont, dans le contexte actuel, la voie la plus sûre pour que les entreprises disposent des financements qui leur sont nécessaires.
Ce document est protégé par la loi sur le droit d'auteur. L'utilisation des services d'Érudit (y compris la reproduction) est assujettie à sa politique d'utilisation que vous pouvez consulter en ligne.

https://apropos.erudit.org/fr/usagers/politique-dutilisation/ 


\title{
Financement des entreprises et évolution du système financier ${ }^{1}$
}

\author{
Henri JULLIEN* \\ Bernard PARANQUE** \\ Banque de France
}

\section{MOTS CLÉS}

\section{Financement - Marché financier - Accumulation Rentabilité - PMI}

\begin{abstract}
RÉSUMÉ
L'insuffisante capitalisation souvent évoquée à propos des $P M I^{2}$ est en grande partie le résultat d'un effort d'investissement relatif similaire, si ce n'est plus fort, que celui de leurs concurrentes de plus grande taille. Elle est imputable, par ailleurs, à la difficulté d'accès aux marchés financiers pour cette catégorie d'entreprises. Pourtant le gain potentiel escompté d'une participation au capital d'une PMI paraît aussi élevé que celui obtenu dans une entreprise de taille plus grande.

Alors que la période 1985-1990 avait permis de développer toutes les techniques de financement sans contraintes particulières, les années récentes ont été marquées par divers facteurs conduisant les banques et, en règle
\end{abstract}

* Henri Jullien est adjoint au directeur des entreprises de la Banque de France. Il intervient lors de séminaires consacrés à l'évolution des marchés financiers et aux problèmes de financement que rencontrent les entreprises. Adresse: Banque de France. Direction des entreprises - cc : $36-1363$, 39, rue Croix des Petits Champs, 75049 Paris, cedex 01.

** Bernard Paranque est économiste à la Direction des entreprises (Observatoire des entreprises - Centrale de bilans). Il conduit des recherches sur la situation économique et financière des entreprises, particulièrement des PMI. Il est l'auteur de différentes études sur le sujet publiées dans la collection «Entreprise» de la Banque de France : «Compétitivité et rentabilité des entreprises industrielles», "Localisation et performances: la situation des entreprises régionales en 1992 », «Emploi, accumulation et rentabilité financière», "Investissement productif et croissance externe» (en collaboration). Adresse: Banque de France. Direction des entreprises cc: 44-1356, 39, rue Croix des Petits Champs, 75049 Paris, cedex 01.

1. Présenté aux $11^{\mathrm{c}}$ Journées internationales d'économie monétaire et bancaire à Nice les 9 et 10 juin 1994 et actualisé pour la présente publication.

2. Hors la période de leur création et le cas des entreprises de moins de 20 salariés, qui revêtent un caractère particulier concernant l'apport initial et le financement. 
générale, tous les opérateurs financiers à revenir largement sur des pratiques libérales qui avaient facilité l'accès des entreprises aux marchés des capitaux.

Face à la montée des risques et à la dégradation des résultats, les banques se sont montrées particulièrement sélectives vis-à-vis des PME. Soit elles ont largement supprimé les financements de secteurs considérés comme sinistrés (immobilier, BTP, informatique...), soit elles n'accordent des crédits qu'avec prudence.

Si l'analyse statistique fait ressortir des situations grandes entreprises / PMI relativement comparables, le constat pour les années récentes montre dans la réalité un niveau de risque sensiblement supérieur.

Par conséquent, au-delà d'un niveau raisonnable de fonds propres que le marché doit pouvoir fournir, la restauration d'un véritable partenariat basé sur la confiance entre banques et entreprises et le partage des risques dans le cadre d'un groupement financier sont, dans le contexte actuel, la voie la plus sûre pour que les entreprises disposent des financements qui leur sont nécessaires.

\section{ABSTRACT}

Inadequate capitalization of small and medium-sized industrial enterprises ${ }^{3}$ is a frequently mentioned phenomenon. It is largely the result of their investment effort, which is, relatively speaking, similar or greater than that of their larger competitors. This trend can also be attributed to the difficulty small firms have in gaining access to capital markets. And yet the potential gain to be expected from an interest in a small industrial enterprise seems to be as great as that from a larger firm.

In the years from 1985 to 1990 , all types of financing techniques were able to develop without any particular constraints. However, in recent years, various factors have emerged to cause banks, and financial operators in general, to abandon many of the liberal practices that had given businesses easier access to financial markets.

Rising risks and falling profits have made the banks particularly selective in their dealings with small businesses. Either they have cut off most of their lending to sectors deemed to be in trouble, such as real estate, construction and data processing, or they take great care in giving loans.

Statistical analysis shows that large firms and small businesses are in fairly comparable situations, but the figures for recent years show that the actual level of risk is significantly higher.

As a result, the current context makes a new partnership built on trust between banks and businesses, along with the pooling of risks, the best way of ensuring that businesses get the financing they need, in addition to a reasonable amount of equity that they should be to raise on the market.

3. This does not apply to companies in the start-up period and those with fewer than 20 employees, which have distinctive characteristics concerning the initial capital contribution and financing. 


\section{RESUMEN}

La insufficiente capitalización frecuentemente mencionada con respecto a las PyMIs $^{4}$ (pequeñas y medianas industrias), se debe en gran parte a un esfuerzo relativo de inversión que es comparable o mayor que el de sus competidores de más envergadura. Por otra parte, se la puede atribuir al difícil acceso que tiene esta categoría de empresas a los mercados financieros. Sin embargo, el beneficio potencial esperado de una participación al capital de una PyMI parece ser tan elevado como el que se puede obtener en una empresa de mayor tamaño.

Mientras que el período 85-90 había permitido desarrollar todas las técnicas de financiación sin restricciones particulares, los años recientes se han caracterizado por diversos dactores que han llevado a los bancos, y por regla general a todos los operadores financieros, a un decidido cuestionamiento de prácticas liberales que habian facilitado el acceso de las empresas a los mercados de capitales.

Ante la agravación de los riesgos y el empeoramiento de los resultados, los bancos se volvieron particularmente selectivos hacia las PyMEs. O bien han suprimido de manera generalizada la financiación de sectores considerados damnificados (inmobiliario, construcción y obras públicas, informática, etc.), o sólo otorgan créditos con cautela.

Si bien el análisis estadístico pone de relieve situaciones de grandes empresas y de PyMls que son relativamente comparables, el saldo de los años recientes muestra que en la realidad, el nivel de riesgo es sustancialmente superior.

Por consiguiente, por encima de un nivel razonable de fondos propios que el mercado debe poder proporcionar, la restauración de una auténtica colaboración, basada en la confianza, entre bancos y empresas, y la asunción compartida de riesgos en el marco de un pool constituyen, en el contexto actual, el método más seguro que permita a las empresas disponer de las finanzas necesarias.

\section{Introduction}

Divers travaux ont pu montrer qu'il n'existait pas de relation positive entre l'importance des fonds propres et le niveau de performances des entreprises. En fait, les firmes les plus dynamiques en matière d'investissement ou d'emploi sont souvent celles qui sont les plus endettées. Toutefois, cette appréciation bute sur le diagnostic: en cas de disparition d'un client important ou de récession

4. Exceptuando el período de su creación y el caso de empresas de menos de 20 asalariados, que revisten un carácter particular en materia de aportación inicial y financiación. 
des marchés, ces mêmes entreprises sont celles qui sont alors les moins à même de pouvoir absorber ce retournement de situation. La hausse des défaillances depuis 1990-1992 est là pour souligner l'enjeu du nécessaire équilibre entre les financements et le niveau des fonds propres.

Par ailleurs, on peut s'interroger sur le développement des marchés financiers et la déréglementation qui l'a accompagné, particulièrement à l'égard des PME. La possibilité théorique d'accéder directement aux ressources et l'incapacité pratique dans laquelle ces sociétés se trouvent pour le faire, conduisent à analyser la place et le rôle du système bancaire à leur égard. Ce dernier est à l'interface de la sphère réelle et de la sphère financière. C'est donc lui qui va influencer les choix de financement afin d'assurer «l'équilibre du système». Mais, par voie de conséquence, ce sont les PME qui doivent gérer les conséquences de ces décisions, que ce soit en termes de volume de crédits ou de taux d'intérêts.

\section{Fonds propres et rentabilité: les PMI sont-elles handicapées par leur dynamisme?}

Pour consolider ou accroître son autonomie financière ${ }^{5}$, une entreprise peut faire appel à ses actionnaires ou dégager un résultat qui sera capitalisé. Cela implique, pour être réalisable, que la firme ait facilement accès au marché financier (ou que ses principaux actionnaires puissent lever aisément des fonds) ou que ses performances économiques et financières soient suffisamment élevées. Toutefois, l'intensité de l'autonomie financière d'une entreprise, mesurée par la valeur de ses fonds propres, n'est pas pour autant synonyme de performances économiques supérieures et, par voie de conséquence, n'est pas totalement pertinente pour évaluer la santé de la firme.

Dans l'industrie, au début des années 1980 , la rentabilité financière brute des $\mathrm{PMI}^{6}$, en particulier des plus petites, était supérieure à celle de leurs concurrentes de plus grande taille (Bardos et Cordier, 1991). À compter de 1986, l'opposition s'est sensiblement atténuée puisque les grandes entreprises, grâce essentiellement à une diminution de la part des charges salariales dans la valeur ajoutée (Vieille, 1992; Artus,1992), ont fortement amélioré leur rentabilité financière brute. Le redressement a été nettement moins important dans les petites PMI, en raison principalement d'un partage de la valeur ajoutée en

5. À savoir le poids de ses dettes financières par rapport à ses fonds propres.

6. Entreprises de l'industrie occupant entre 20 et 500 salariés. 
faveur de l'entreprise moins favorable et d'un endettement plus élevé7. Toutefois, l'emploi y a été plus favorisé que dans les entreprises de plus grande taille ${ }^{8}$.

Dès lors, si l'appréciation bilantielle de la situation financière des PMI peut inciter à croire à une insuffisante capitalisation de leur part, l'analyse de leur comportement économique et financier indique, quant à elle, des rentabilités économiques et financières comparables, voire supérieures, à celles des grandes entreprises. L'insuffisante capitalisation souvent évoquée à propos des $\mathrm{PMI}^{9}$ est alors en grande partie le résultat d'un effort d'investissement relatif similaire, si ce n'est plus fort, à celui de leurs concurrentes de plus grande taille. Celui-ci se traduit par des charges de maintien du potentiel productif plus élevées (dotations aux amortissements) grevant leur résultat. Elle est imputable, par ailleurs, à la difficulté d'accès aux marchés financiers pour cette catégorie d'entreprises. Autrement dit, le gain potentiel escompté d'une participation au capital d'une PMI paraît aussi élevé que celui obtenu dans une entreprise de taille plus grande. Toutefois, la réalisation de ce gain et la valeur de la capitalisation vont dépendre, d'une part, de la survie de l'entreprise et, d'autre part, des investissements engagés qui déterminent la réalisation des amortissements à effectuer et de la politique de distribution de dividendes suivie.

\subsection{Les fonds propres garantissent la solvabilité de l'entreprise mais non sa rentabilité...}

\subsubsection{De la nécessité des fonds propres...}

Les fonds propres ont pour fonction cardinale de garantir la solvabilité de l'entreprise. Ils constituent, pour une firme et pour les agents économiques qui y sont engagés, une «réserve» de sécurité permettant de faire face aux accidents d'origine interne ou externe. Par exemple, en période de ralentissement conjoncturel, les entreprises peu endettées sont avantagées par rapport à celles qui ont privilégié l'emprunt au détriment des fonds propres. Les premières peuvent étaler dans le temps la rémunération de leurs apporteurs en capitaux externes,

7. Voir le dossier «Crise, comment en sortir? », paru dans l'Expansion du 4-17 mars 1993, en particulier les articles de Jean Boissonnat, Jacques Barraux et Michel Aglietta.

8. Voir les travaux présentés à l'occasion de la $18^{\mathrm{e}}$ Journée des Centrales de Bilans, Paris, novembre 1993.

9. Hors la période de leur création et le cas des entreprises de moins de 20 salariés, qui revêtent un caractère particulier concernant l'apport initial et le financement. 
alors que les secondes doivent faire face à des échéances obligatoires. Autrement dit, les fonds propres peuvent se définir à l'aide d'une triple condition (Manceau, 1992):

- les fonds propres sont des capitaux à exigibilité nulle ;

- les fonds propres sont des ressources qui ne peuvent prétendre à une rémunération fixe ;

- les fonds propres ont pour fonction ultime de couvrir le risque de l'entreprise.

Si la détention par une entreprise d'un minimum de fonds propres apparaît vitale, il est, par contre, difficile de définir dans l'absolu un partage optimal entre fonds propres et endettement. En fait, chaque entreprise, en fonction de sa situation économique et commerciale, cherchera à optimiser le recours au financement externe selon le coût de celui-ci, mais aussi en fonction du degré d'autonomie qu'elle veut ou peut sauvegarder et de ses projets de développement.

À toute stratégie industrielle, qu'elle soit de croissance interne ou externe, correspond donc une stratégie financière. Celle-ci traduit le mode de financement retenu, c'est-à-dire le choix entre autofinancement, recours à l'endettement ou appel aux actionnaires. La multitude des arbitrages possibles entre ces différentes sources de financement induit des comportements qui varient d'une firme à l'autre.

Cette question d'un niveau optimal de fonds propres n'a d'ailleurs cessé d'être discutée tout au long des trente dernières années, depuis le théorème d'indifférence de Modigliani-Miller ${ }^{10}$ (1958) selon lequel la valeur de marché d'une entreprise est indépendante du partage de son passif entre endettement et fonds propres. Ce théorème n'est en fait vérifié que sous des conditions adéquates de fonctionnement des marchés financiers «parfaits». En particulier, il faut que la détention de titres de créances puisse être librement arbitrée avec celle des droits de propriété que sont les actions. Selon ce théorème, la valeur de marché des firmes est indifférente à la structure du passif de leur bilan (appel aux actionnaires ou recours à l'endettement). Dans ce cas, un investissement productif n'est réalisé que s'il augmente la valeur de marché de l'entreprise, ce qui ne dépend que de la comparaison entre son rendement spécifique et le coût moyen du capital. La décision est alors indépendante de la structure de financement retenue (De Bandt et Jacquinot, 1991).

10. «The cost of capital, corporate finance and the theory of investment », American Economic Review, mai 1958. 
Or, les marchés ne sont pas parfaits, comme le montre l'existence d'asymétrie d'information ${ }^{11}$ et de risques (entre autres de faillite) liés à la perte de maîtrise de la charge de la dette, ce qui peut conduire le prêteur soit à exiger des garanties supplémentaires, soit à rationner le crédit (par les taux ou les quantités). Par ailleurs, l'accès à ces marchés est limité à un faible nombre des PMI.

Dans ce contexte, les fonds propres des entreprises dépendent étroitement du montant de l'épargne à long terme en France (Icard, 1991) mais aussi, voire surtout pour les PMI, des possibilités d'accès à cette épargne. Pour une firme donnée, ils sont donc étroitement liés à sa rentabilité financière (Paranque, 1992), puisque les bénéfices dégagés peuvent être capitalisés ou permettre d'offrir une rémunération attrayante pour les futurs associés.

Toutefois, il ne semble pas y avoir de liaisons positives entre le degré d'autonomie financière et les performances économiques ${ }^{12}$ des entreprises (Paranque, 1994a) ${ }^{13}$. En revanche, l'autonomie d'une entreprise décroît avec l'intensité de son effort d'investissement, en raison des besoins de financement qu'il suscite.

\subsubsection{Un effort de renouvellement du potentiel productif et des rentabilités plus élevés dans les PMI ${ }^{14}$}

Les PME présentent une combinaison productive moins capitalistique que les grandes entreprises (GE), mais leur effort d'accroissement du potentiel productif, mesuré par le taux d'accumulation (investissement sur capital productif), a cependant été de 1985 à 1991 supérieur à celui de leur homologue de plus grande taille. Le taux d'accumulation est plus pertinent que le seul taux

11. L'asymétrie d'information signifie que tous les agents économiques ne disposent pas de toute l'information. Les dirigeants de sociétés ont ainsi, du fait de leur situation, une information privilégiée sur la situation et les perspectives de développement économique et financier de la firme. Cette asymétrie explique que dans certaines circonstances, le recours à des contrats de dettes soit privilégié. Il peut en être ainsi, par exemple, pour le financement des besoins transitoires. En effet, «si l'on considère que les dirigeants protègent les intérêts des seuls anciens actionnaires », une émission d'actions sera mal accueillie. À la limite, elle pourrait signifier aux yeux d'éventuels investisseurs que le cours atteint par les actions est jugé trop élevé par les dirigeants. Les nouveaux actionnaires exigent donc une prime de risque considérable, ce qui favorise les autres formes de financement (De Bandt et Jacquinot, 1991).

12. Rentabilités économique et financière.

13. Ce constat dépend fortement du type d'indicateur retenu, voir infra et Paranque (1994b).

14. $C f$. le lexique, en fin d'article, pour une définition des composants des ratios. 
d'investissement (investissement sur valeur ajoutée) dans la mesure où il tient compte de l'efficacité de la combinaison productive, moindre dans les firmes de taille importante, et de l'évolution différenciée selon la taille de la substitution capital sur travail, plus forte dans les grandes entreprises. En effet, le montant de valeur ajoutée consacré à l'investissement est plus faible dans les PMI que dans les firmes de taille plus importante. Cette différence d'appréciation de l'effort de renouvellement du potentiel productif s'explique par une moindre efficacité du capital dans les grandes entreprises. Autrement dit, la création de valeur ajoutée par unité de capital semble y être relativement insuffisante ${ }^{15}$ en regard des investissements réalisés.

Cette opposition sur les comportements d'investissement a comme conséquence des besoins de financement élevés qui, joints aux difficultés d'accès aux marchés financiers (problèmes techniques, moyens à mettre en œuvre, temps, etc.) induisent, dans les PMI, un taux d'endettement (y compris les prêts des groupes et des associés) plus élevé que dans les grandes entreprises, bien que les taux d'intérêt apparents y soient en permanence moins favorables. Toutefois, si l'autonomie financière ${ }^{16}$ des PMI est moindre, comparée à celle de leurs concurrentes de plus grande taille à compter de 1984-1985, cela tient surtout aux restructurations importantes intervenues dans nombre de grandes entreprises afin de réduire leur endettement et de restaurer leur santé financière. Ainsi, sur la décennie 1980, le taux d'endettement des entreprises de moins de 500 personnes est relativement stable (Paranque, 1992).

\subsection{Les PMI sont-elles handicapées par leur dynamisme ${ }^{17}$ ?}

L'objet de cette section est autant de donner quelques résultats sur l'exercice 1992 que d'attirer l'attention du lecteur sur la nécessaire cohérence entre la définition des objectifs de l'analyse et l'élaboration d'indicateurs adaptés à ces objectifs. En effet, cette exigence de cohérence est indispensable à l'identification des différents niveaux d'interprétation possibles et donc à la pertinence du diagnostic porté sur la situation financière des entreprises.

15. Suivant une formule comptable, $\frac{I}{C}=\frac{I}{V A} \times \frac{V A}{C}$, nous aurions le taux d'accumulation, le taux d'investissement et l'efficacité du capital.

16. Mesurée ici par le rapport «apports externes sur financement propre».

17. Les entreprises industrielles de moins de 500 salariés sont comparées aux firmes de plus grande taille sur la période 1991-1993. Le taux de couverture de l'échantillon est de $56 \%$ des effectifs salariés du fichier exhaustif de l'Institut national de statistique et d'étude économique (INSEE) des entreprises assujetties au régime du bénéfice industriel et commercial. Il est de $77 \%$ pour les entreprises de plus de 500 salariés, lesquelles représentent $1,3 \%$ des firmes du fichier INSEE contre 7,3\% pour le présent échantillon. 


\subsubsection{Une moindre autonomie des $P M{ }^{18} \ldots$}

L'autonomie financière est déterminée par le degré d'indépendance de l'entreprise par rapport à son environnement financier. Deux indicateurs sont calculés :

- l'un en brut, issu de la méthodologie de la Centrale de Bilans de la Banque de France, financement propre sur capital engagé : capitaux propres de la liasse fiscale plus les réserves d'autofinancement constitués par les amortissements cumulés, rapportés au capital engagé, c'est-àdire le financement propre, plus les dettes financières y compris celles contractées auprès des groupes et associés;

- l'autre en net, plus souvent utilisé, capitaux propres sur total du bilan.

\section{TABLEAU 1 \\ L'autonomie financière \\ (en \%)}

\begin{tabular}{lcccccc}
\hline 1992 & \multicolumn{3}{c}{ PMI } & \multicolumn{3}{c}{ GE } \\
\hline & $\begin{array}{c}\text { Borne } \\
\text { inf. }\end{array}$ & Moyenne & $\begin{array}{c}\text { Borne } \\
\text { sup. }\end{array}$ & $\begin{array}{c}\text { Borne } \\
\text { inf. }\end{array}$ & Moyenne & $\begin{array}{c}\text { Borne } \\
\text { sup. }\end{array}$ \\
\hline $\begin{array}{l}\text { Financement propre/capital } \\
\text { engagé }\end{array}$ & 68,3 & 68,7 & 69,2 & 73,9 & 75,6 & 77,3 \\
Capitaux propres/bilan & 31,5 & 32,1 & 32,6 & 33,8 & 36,0 & 38,2 \\
\hline
\end{tabular}

Source et réalisation : Banque de France - Observatoire des entreprises

Tél. : +33 (1)42925658. Mise à jour février 1994.

\begin{tabular}{lcccccc}
\hline 1993 & \multicolumn{3}{c}{ PMI } & \multicolumn{3}{c}{ GE } \\
\hline & $\begin{array}{c}\text { Borne } \\
\text { inf. }\end{array}$ & Moyenne & $\begin{array}{c}\text { Borne } \\
\text { sup. }\end{array}$ & $\begin{array}{c}\text { Borne } \\
\text { inf. }\end{array}$ & Moyenne & $\begin{array}{c}\text { Borne } \\
\text { sup. }\end{array}$ \\
\hline $\begin{array}{l}\text { Financement propre/capital } \\
\text { engagé }\end{array}$ & & & & & & \\
Capitaux propres/bilan & 37,6 & 72,0 & 72,4 & 75,2 & 76,7 & 78,3 \\
\hline
\end{tabular}

Source et réalisation : Banque de France - Observatoire des entreprises

Tél. : +33 (1)42925658. Mise à jour octobre 1994.

18. Les tests d'inégalité de moyenne permettent de définir quand l'écart constaté est statistiquement discernable. L'intervalle de confiance présenté (borne inférieure et borne supérieure) est calculé au seuil de $5 \%$. Pour s'assurer que le constat ne dépend pas de l'hypothèse de loi normale, un test non paramétrique a été réalisé sur la médiane. Il n'est évoqué que lorsqu'il contredit le résultat précédent. 
En brut, c'est-à-dire en tenant compte des réserves d'autofinancement constituées par les amortissements cumulés, l'autonomie financière des PMI est inférieure à celle des grandes entreprises. Cet écart s'explique, pour l'essentiel, par les différences existant dans l'effort de renouvellement du potentiel productif (mesuré par le taux d'accumulation). Il entraîne en effet des besoins de financement d'autant plus grand que, compte tenu des relations particulières des PMI avec les grandes entreprises et de la spécificité de leur insertion dans le système productif, les entreprises de moins de 500 salariés assument des besoins en fonds de roulement d'exploitation plus élevé que leurs concurrentes de plus grande taille.

Le recours à l'endettement est, en outre, d'autant plus nécessaire que les PMI ont plus de difficultés à accéder aux marchés financiers pour y mobiliser des ressources propres. Ce handicap apparaît quand on évalue leur autonomie en net par le ratio "capitaux propres sur bilan» (fournisseurs inclus): elle est toujours moindre que celle des firmes de plus grande taille.

Cette dynamique particulière des PMI en matière d'investissement ne semble toutefois pas les handicaper pour ce qui est des performances économiques et financières.

\subsection{2. ... mais des rentabilités similaires, voire plus élevées, dans les entreprises de moins de 500 salariés}

Trois ratios sont étudiés ${ }^{19}$ :

- la rentabilité brute globale: «l'excédent brut global sur le capital engagé », à savoir l'excédent brut d'exploitation plus les produits et charges exceptionnelles deviennent des indicateurs d'une contrainte de rentabilité générale ${ }^{20}$;

- la rentabilité financière brute : «la capacité d'autofinancement brute sur le financement propre » exprime une contrainte de rentabilité à l'égard des actionnaires hors politique d'investissement ;

- la rentabilité financière nette des charges de maintien du potentiel productif précise la contrainte précédente en intégrant la politique d'investissement, mais avant les choix de rémunération des actionnaires.

19. Voir lexique pour plus de détails.

20. Elle résulte de l'efficacité du capital et du taux de marge. De 1985 à 1991, on constate une baisse de l'efficacité du capital engagé et un redressement du taux de marge particulièrement sensible dans les grandes entreprises traduisant une restauration du partage de la valeur ajoutée favorable à la rémunération des capitaux. 


\section{TABLEAU 2}

\section{Les rentabilités}

(en \%)

\begin{tabular}{lrrrrrr}
\hline \multicolumn{1}{c}{1992} & \multicolumn{3}{c}{ PMI } & \multicolumn{3}{c}{ GE } \\
\hline & $\begin{array}{c}\text { Borne } \\
\text { inf. }\end{array}$ & Moyenne & $\begin{array}{c}\text { Borne } \\
\text { sup. }\end{array}$ & $\begin{array}{c}\text { Borne } \\
\text { inf. }\end{array}$ & Moyenne & $\begin{array}{c}\text { Borne } \\
\text { sup. }\end{array}$ \\
\hline Rentabilité brute globale* & 13,2 & 13,5 & 13,7 & 11,6 & 12,6 & 13,5 \\
Rentabilité financière brute* & 11,7 & 12,0 & 12,3 & 9,8 & 11,0 & 12,2 \\
Rentabilité financière nette & $-0,2$ & 0,2 & 0,6 & 1,5 & 3,0 & 4,6 \\
\hline
\end{tabular}

Source et réalisation : Banque de France - Observatoire des entreprises Tél. : +33 (1)42925658. Mise à jour février 1994.

\begin{tabular}{lrrrrrr}
\hline \multicolumn{1}{c}{1993} & \multicolumn{3}{c}{ PMI } & \multicolumn{3}{c}{ GE } \\
\hline & $\begin{array}{c}\text { Borne } \\
\text { inf. }\end{array}$ & Moyenne & $\begin{array}{c}\text { Borne } \\
\text { sup. }\end{array}$ & $\begin{array}{c}\text { Borne } \\
\text { inf. }\end{array}$ & Moyenne & $\begin{array}{c}\text { Borne } \\
\text { sup. }\end{array}$ \\
\hline Rentabilité brute globale* & 11,3 & 11,6 & 11,8 & 9,3 & 10,3 & 11,3 \\
Rentabilité financière brute* & 9,6 & 9,9 & 10,3 & 7,3 & 8,5 & 9,7 \\
Rentabilité financière nette & $-0,3$ & 0,1 & 0,5 & $-0,15$ & 1,3 & 2,8 \\
\hline
\end{tabular}

Source et réalisation : Banque de France - Observatoire des entreprises Tél. : +33 (1)42 925658 . Mise à jour octobre 1994.

* Le test sur la médiane indique un écart statistiquement discernable, 50,5\% des PMI ayant un ratio supérieur à la médiane contre $41,8 \%$ pour les grandes entreprises. L'écart est plus net sur la rentabilité financière brute avant 1992, voir «Emploi, accumulation et rentabilité financière», étude citée; en 1991, elle est respectivement de $14,7 \%$ et de $12,7 \%$.

Leur moindre autonomie financière n'empêche pas les PMI de dégager une rentabilité brute globale le plus souvent supérieure à celle des grandes entreprises. Ces performances s'expliquent alors, pour une large part, par une efficacité du capital plus élevée dans les PMI que dans les grandes entreprises, laquelle efficacité fait plus que compenser un taux de marge quelque peu inférieur ${ }^{21}$. Cette hiérarchie se retrouve sur la rentabilité financière brute qui s'est accrue dans toutes les firmes jusqu'en 1989 avant de se replier dans les grandes entreprises dès 1990 puis, y compris dans les PMI, en 1991 (Paranque, 1994a).

21. Autrement dit, le partage de la valeur ajoutée est plus favorable à la rémunération des capitaux dans les grandes entreprises que dans les PMI, ce qui est cohérent avec la moindre intensité capitalistique de ces dernières. 
Si la rentabilité financière nette ${ }^{22}$ est alors plus faible dans les PMI que dans les entreprises plus importantes, cela résulte d'un effort d'investissement plus soutenu qui génère des dotations aux amortissements rapportés au financement propre plus élevées ${ }^{23}(11,6 \%$ contre $7,8 \%$ dans les grandes entreprises en 1992).

Si l'on déduit les dividendes versés de la capacité d'autofinancement brut, on obtient l'autofinancement. Rapporté au financement propre, il constitue un indicateur de rentabilité après rémunération des actionnaires et donc une évaluation de la capacité de capitalisation de l'entreprise, avant prise en compte de la politique d'investissement.

TABLEAU 3

La capitalisation

(en \%)

\begin{tabular}{lcccccc}
\hline \multicolumn{1}{c}{1992} & \multicolumn{3}{c}{ PMI } & \multicolumn{3}{c}{ GE } \\
\hline & $\begin{array}{c}\text { Borne } \\
\text { inf. }\end{array}$ & Moyenne & $\begin{array}{c}\text { Borne } \\
\text { sup. }\end{array}$ & $\begin{array}{c}\text { Borne } \\
\text { inf. }\end{array}$ & Moyenne & $\begin{array}{c}\text { Borne } \\
\text { sup. }\end{array}$ \\
\hline $\begin{array}{l}\text { Autofinancement/ } \\
\text { financement propre* }\end{array}$ & 9,9 & 10,3 & 10,6 & 7,3 & 8,7 & 10,2 \\
$\begin{array}{l}\text { Autofinancement net/ } \\
\text { financement propre }\end{array}$ & $-1,0$ & $-0,7$ & $-0,3$ & $-0,6$ & 0,8 & 2,2 \\
\hline
\end{tabular}

Source et réalisation : Banque de France - Observatoire des entreprises Tél. : +33 (1)42925658. Mise à jour février 1994.

\begin{tabular}{lcccccc}
\hline 1993 & \multicolumn{3}{c}{ PMI } & \multicolumn{3}{c}{ GE } \\
\hline & $\begin{array}{c}\text { Borne } \\
\text { inf. }\end{array}$ & Moyenne & $\begin{array}{c}\text { Borne } \\
\text { sup. }\end{array}$ & $\begin{array}{c}\text { Borne } \\
\text { inf. }\end{array}$ & $\begin{array}{c}\text { Moyenne } \\
\text { sup. }\end{array}$ \\
\hline $\begin{array}{l}\text { Autofinancement/ } \\
\text { financement propre }\end{array}$ & 7,0 & 7,4 & 7,8 & 4,0 & 5,4 & 6,8 \\
\hline
\end{tabular}

Source et réalisation : Banque de France - Observatoire des entreprises

Tél. : +33 (1)42925658. Mise à jour octobre 1994.

* Le test sur la médiane montre un écart statistiquement discernable au seuil de $5 \%$ en faveur des PMI $(50,7 \%$ contre $38,6 \%)$.

22. En calculant un ratio capacité d'autofinancement net sur capitaux propres, et non plus rapporté au financement propre (qui est brut), le constat est similaire.

23. Le poids des amortissements dans le financement propre est de $51 \%$ dans les PMI et de $42,5 \%$ dans les entreprises de plus de 500 salariés. 
Les PMI sont toujours plus rentables que les grandes entreprises après paiement de dividendes aux actionnaires. L'écart s'accroît quelque peu d'une catégorie de taille à l'autre quand on passe de la rentabilité financière brute au ratio «autofinancement sur financement propre» (de 1 point à 1,6 point). Le poids des dividendes est en effet plus élevé dans les grandes entreprises que dans les PMI (respectivement 2,5\% du financement propre contre $1,7 \%$ et $18,5 \%$ de la capacité d'autofinancement brute contre $11 \%$ ).

En revanche, après déduction des charges de maintien du potentiel productif, et donc en net, les situations sont quelque peu différentes (mais plus favorables en 1992 aux PMI sur le ratio «autofinancement net sur capitaux propres $\left.{ }^{24} »\right)$. Autrement dit, le poids des dotations aux amortissements est supérieur dans les PMI alors que celui des dividendes est moindre. Le constat est symétrique pour les grandes entreprises. Ainsi, la capacité de capitalisation des résultats, et donc de renforcement des capitaux propres, n'est que peu différente d'une catégorie de taille à l'autre.

Ce constat est une illustration de logique économique différente, l'une plus industrielle, l'autre plus financière. Il apparaît bien une contrainte de rentabilité spécifique dans les grandes firmes à l'égard des actionnaires relative à l'intensité des liens avec le marché financier: la rentabilité financière nette et la rémunération des actionnaires y sont plus élevées.

\section{TABLEAU 4}

\section{La distribution}

(en \%)

\begin{tabular}{ccccccc}
\hline 1992 & \multicolumn{3}{c}{ PMI } & \multicolumn{3}{c}{ GE } \\
\hline & $\begin{array}{c}\text { Borne } \\
\text { inf. }\end{array}$ & Moyenne & $\begin{array}{c}\text { Borne } \\
\text { sup. }\end{array}$ & $\begin{array}{c}\text { Borne } \\
\text { inf. }\end{array}$ & $\begin{array}{c}\text { Moyenne } \\
\text { sup. }\end{array}$ \\
\hline Dividendes / capitaux propres & 3,0 & 3,1 & 3,3 & 4,2 & 4,7 & 5,3 \\
\hline
\end{tabular}

Source et réalisation: Banque de France - Observatoire des entreprises Tél. : +33 (1)42 925658 . Mise à jour février 1994.

\begin{tabular}{ccccccc}
\hline 1993 & \multicolumn{3}{c}{ PMI } & \multicolumn{3}{c}{ GE } \\
\hline & $\begin{array}{c}\text { Borne } \\
\text { inf. }\end{array}$ & Moyenne & $\begin{array}{c}\text { Borne } \\
\text { sup. }\end{array}$ & $\begin{array}{c}\text { Borne } \\
\text { inf. }\end{array}$ & $\begin{array}{c}\text { Moyenne } \\
\text { Borne } \\
\text { sup. }\end{array}$ \\
\hline Dividendes/capitaux propres & 3,8 & 4,0 & 4,3 & 4,0 & 5,3 & 6,1 \\
\hline
\end{tabular}

Source et réalisation : Banque de France - Observatoire des entreprises Tél. : +33 (1)42 925658 . Mise à jour octobre 1994.

24. D'après le test sur la médiane. 
L'opposition constante entre les entreprises selon leur taille souligne la spécificité de la contrainte de rentabilité plus marquée à l'égard des prêteurs dans les PMI. La rentabilité économique doit y être meilleure pour assurer la charge de la dette et le poids de la politique d'investissement.

Il faudrait préciser cette opposition par une étude complète de l'incidence de la fiscalité sur la politique d'amortissement, mais cela dépasse le champ du présent travail. Il n'en reste pas moins que l'amortissement dégressif réduit, sur le plan comptable, le délai de retour sur investissement et peut donc amplifier les effets de la politique d'investissement proprement dite sur le résultat.

\section{Fonds propres et rentabilité : la mutation financière récente a-t-elle favorisé les PMI?}

Des structures en capitaux légèrement inférieures dans les PMI et un effort d'investissement égal sinon plus grand, tel est le constat de base de cette analyse, positif si l'on se place dans la position d'un investisseur ou prêteur estimant la trajectoire à venir d'une entreprise. De plus, ce constat statique est bien complété par l'analyse des niveaux de rentabilité et d'autofinancement assez peu différents. Certes, les dividendes rapportés aux capitaux propres sont plus faibles dans les PMI, mais est-ce cela que recherche un investisseur?

Ces constats pourraient laisser penser que les niveaux d'investissement plus élevés dans les PMI sont gages de taux de capitalisation, et donc de gains en capital, intéressants pour l'avenir, et de nature à attirer les investisseurs.

Le dynamisme des PMI serait-il alors porteur d'un tel niveau de risque que tous les fournisseurs de capitaux s'en tiennent à distance respectable? Avant la mutation financière, il était habituel de mettre en cause l'inadéquation des marchés financiers de l'époque aux spécificités des PMI. Celle-ci s'est produite et, avec le recul, il est possible de faire le point sur les évolutions intervenues depuis 1986.

\subsection{La mutation financière récente a-t-elle entraîné des évolutions dans le financement des PMI?}

À partir de 1984, la France a entrepris sa mutation financière, suivant en cela la voie ouverte au début des années 1980 par les pays anglo-saxons. Compte tenu des structures financières figées et très réglementées de l'époque (en particulier encadrement du crédit, contrôle des changes, cloisonnement des intervenants), ce mouvement ne pouvait être que de grande ampleur et brutal, aboutissant à un bouleversement complet du paysage financier français. Ce 
phénomène intervenant dans une situation conjoncturelle faste, marquée entre autres par des taux d'investissement élevés, s'est mis en place dans les meilleures conditions, permettant aux opérateurs, tant banquiers qu'entreprises, de modifier leurs organisations et de s'adapter au mieux au nouveau contexte. La fin des années 1980 pouvait donc donner en France l'apparence d'un monde idéal dans le domaine de la finance, notamment dans les relations entre les banques et les entreprises. Nous verrons cependant qu'au-delà des grandes entreprises tous les problèmes étaient loin d'être résolus pour les entreprises de taille réduite. Dans ce contexte, le retournement conjoncturel récent a approfondi l'écart entre les grandes et les petites entreprises et fait resurgir des difficultés dans les relations entre les entreprises et le monde financier.

\subsubsection{La déréglementation financière à partir des années 1985 en France}

Un bref rappel des principales modifications intervenues s'avère indispensable pour mesurer l'ampleur de la mutation financière intervenue et, surtout, pour en apprécier les conséquences sur l'action des banques et des entreprises.

1983: Ouverture des possibilités de placement des entreprises:

Obligations à bons de souscription d'actions, certificats d'investissements, titres participatifs, création du second marché boursier accessible aux petites entreprises.

1984: Suppression de l'encadrement du crédit et nouvelle loi bancaire donnant plus de souplesse à l'action des établissements.

1985: Création des certificats de dépôt, titres de créance négociables ouverts aux banques, suivi des billets de trésorerie destinés aux entreprises et d'autres instruments négociables.

1985: Création du marché à terme des instruments financiers (MATIF).

1986: Large assouplissement du contrôle des changes.

Au terme de ces quatre années d'évolution accélérée, le panorama financier français se trouvait bouleversé.

- Avec la loi bancaire et la réforme du marché monétaire, le cloisonnement des établissements se trouvait supprimé. La quasi-disparition de toute bonification sur les crédits permettait aux banques à réseau de devenir de véritables banques universelles, étendant leur champ d'activité du court terme au moyen et long terme (concurrençant sur ce terrain les établissements spécialisés) et aux fonds propres des entreprises par l'intermédiaire de filiales spécialisées. Leur rôle de conseil en matière d'ingénierie financière pouvait se développer 
d'autant que l'accès devenu possible aux marchés financiers français et étrangers multipliait leurs possibilités d'actions.

- La suppression du contrôle des changes a permis que se développent en France des produits jusqu'alors inaccessibles aux banques ou entreprises françaises (contrats à terme et options de change), de même que les entreprises ont acquis la liberté de traiter directement avec les opérateurs étrangers (emprunts en devises auprès de banques étrangères, émissions sur marchés de capitaux étrangers (euro commercial paper, TSDI), achats de contrats en devises fermes ou optionnels sur les marchés financiers à travers les banques françaises ou directement auprès des banques étrangères.

- La suppression de l'encadrement du crédit, la réforme du marché monétaire, l'ouverture du marché des billets de trésorerie eurent de multiples conséquences directes ou indirectes sur les possibilités d'action des entreprises.

En termes d'instruments, le marché financier comme celui des crédits se trouvait largement ouvert à tous les intervenants, les conditions de seuil jusqu'alors souvent opposables aux PMI étant, pour la plupart, supprimées.

Cependant, l'analyse montre qu'après une période 1985-1989/1990 très prometteuse, la période récente a mis en évidence les limites de l'ouverture des marchés.

\subsubsection{La période $1985-1989 / 1990$}

Une période conjoncturelle faste permettant le développement de tous les produits issus de la mutation financière et des relations faciles entre les banques et les entreprises grâce à une distribution libérale des crédits.

Au cours de cette période, les entreprises ont montré de réelles velléités d'indépendance à l'égard des banques, les mettant en concurrence avec les marchés financiers tant pour leurs financements que pour leurs placements. D'une situation où le financement correspondait à des crédits bancaires consentis au coup par coup, soit par les banques commerciales (à court terme garantis par des créances commerciales le plus souvent), soit par des établissements spécialisés (moyen terme pour financer des équipements essentiellement), les entreprises se sont retrouvées face à des banques universelles proposant des crédits de toutes durées et développant des formules de globalisation des financements. Cette évolution vers la globalisation sous forme d'avances non garanties ou bénéficiant de garanties simplifiées appelées le plus souvent crédit global d'exploitation anticipant sur l'activité et les bénéfices futurs de l'entreprise était liée tout à la fois à la concurrence des marchés financiers 
(billets de trésorerie en particulier), à la concurrence entre banques qui les poussait à proposer de nouveaux produits et, enfin, à la recherche de réduction de coûts (toute étude de dossier ou gestion de papier commercial remis en garantie étant en effet une charge coûteuse pour les banques). De plus, la contagion des techniques des marchés financiers - appels d'offre en particulier poussait de plus en plus les trésoriers d'entreprises à faire du "shopping» auprès des banquiers de la place, ne retenant que les meilleures offres (moindre coût et facilité de mise en œuvre). La notion de risque bancaire ou de ligne d'engagement s'estompait dans ce cadre derrière une pure notion de trésorerie.

Le fléchissement de l'activité de crédit, en raison de la désintermédiation bancaire, et la concurrence entre établissements avaient entraîné dès 1989 une nette réduction des marges bancaires. Toutefois, en réaction, les banques se sont attachées à améliorer des services que jusqu'alors elles rendaient gratuitement afin de pouvoir en faire accepter la facturation. Elles ont également proposé de nouveaux services (recherches de partenaires, montage d'opérations d'achats d'entreprises, gestion des risques de taux et de change, information économique, etc.), afin de faire progresser la part des commissions dans le produit net bancaire au détriment de la marge d'intérêt.

Enfin, certaines banques ont cherché à restaurer une relation privilégiée avec les entreprises en participant à leur tour de table. Cette voie de l'intervention en fonds propres, typique du modèle allemand et japonais, est cependant loin de faire l'unanimité en France.

Cette description des relations entre banques et entreprises, très favorable aux entreprises, doit toutefois être relativisée lorsque l'on considère les rapports entre les banques et les petites entreprises. En effet, les MOFF (Multi Options Financial Facilities) disparus en 1989, comme les billets de trésorerie ou encore le recours aux opérations sur marchés à terme ou aux options de change, n'ont concerné en France qu'une centaine des plus grands groupes. Il est vrai que la tendance à la centralisation des financements comme de la gestion a multiplié le nombre des sociétés bénéficiant de la mutation financière.

Les petites et moyennes entreprises indépendantes des groupes ont bénéficié de cette période faste, mais dans une plus faible mesure. S'appuyant sur une information financière abondante, elles ont tenté de modifier leurs relations avec les banquiers et cherché à obtenir les mêmes avantages que les grandes. Développant souvent le «shopping» bancaire au détriment de relations de partenariat traditionnel afin de bénéficier de conditions plus favorables basées sur le taux du marché monétaire, elles ont eu tendance en cette période libérale à multiplier leurs sources de crédit (crédit-bail en particulier) et leurs financements, souvent à court terme, même lorsqu'il s'agissait de financer des investissements. 
De surcroît, le développement du second marché et des sociétés de capital-risque ou la possibilité de réaliser des montages «à effet de levier»LBO/RES - pouvaient laisser penser qu'en France toutes les formes d'accès aux capitaux étaient largement ouvertes aux PMI.

\subsubsection{La période actuelle}

\section{Le retour à des relations plus tendues entre banques et entreprises}

Alors que la période 1985-1990 avait permis de développer toutes les techniques sans contraintes particulières, les années récentes ont été marquées par divers facteurs conduisant les banques et, en règle générale, tous les opérateurs financiers à revenir largement sur des pratiques libérales qui avaient facilité l'accès des entreprises aux marchés des capitaux (la réduction des marges, la mise en œuvre du ratio Cooke, le développement en direction des particuliers et dans le domaine immobilier).

Dans ce contexte, les grandes entreprises continuent de bénéficier des évolutions liées à la mutation financière, en ayant pris soin, pour la plupart, de resserrer les liens avec quelques banquiers privilégiés. Ainsi, les MOFF, structures de financement garanties, très ouvertes, ont fait place à des lignes bilatérales «stand-by » négociées le plus souvent à terme (2-5 ans), utilisables à court terme et supérieures aux besoins réels afin de préserver une marge de manœuvre en cas de besoins inattendus. Le recours au «shopping» en faisant le tour des banques pour aller au plus offrant est aujourd'hui réservé à des opérations ponctuelles. Il est vrai que les banques n'ont qu'une marge de manœuvre réduite face à des financements étrangers (avances en devises) ou de marché (billets de trésorerie, BMTN, etc.).

La situation des PME, beaucoup plus dépendante des banques, est en revanche tout autre. Face à la montée des risques et à la dégradation des résultats, les banques se sont montrées particulièrement sélectives. Soit elles ont largement supprimé les financements de secteurs considérés comme sinistrés (immobilier, BTP, informatique, etc.), soit elles n'accordent des crédits qu'avec prudence. Le retour à des principes considérés comme sains - endettement n'excédant pas les capitaux propres, endettement inférieur à 3 ans d'autofinancement, investissements autofinancés à 30 ou $40 \%$, leviers d'endettement dans les opérations de LBO n'excédant pas 2 à 3 ans - ont mis en évidence les excès de la période précédente.

Dans ce contexte, l'analyse des risques de l'entreprise a retrouvé toute son importance et les défauts classiques des PME françaises (relative faiblesse des fonds propres, poids financier de l'endettement, manque de vision de l'avenir) ont été remis en pleine lumière. Toutefois, il s'avère toujours aussi 
difficile de faire prendre conscience à l'entreprise du coût du risque et de l'inclure dans les frais financiers facturés.

Alors que les banquiers parlent de gestion rigoureuse, nombre de PME en France considèrent qu'elles ne trouvent plus en leur banquier l'assistance dont elles ont besoin.

Après une phase d'embellie, la relation s'est donc tendue. Les banquiers reconnaissent que leur offre est plus sélective, mais précisent que les demandes de crédits sont moindres en raison de la crise. Les banquiers regrettent que leurs clients ne souhaitent pas toujours communiquer leurs prévisions à moyen ou long terme, ce qui empêche de négocier des lignes globales d'exploitation. Néanmoins, les banquiers ont besoin de fidéliser leurs clients afin de capturer des mouvements sur l'ensemble de leurs opérations. Globaliser la relation avec l'entreprise est en effet le seul moyen d'améliorer les marges sur tous les services nouveaux qu'ils ont développés.

\section{Deux règles s'imposent donc à eux : restaurer leurs marges en accordant toujours plus d'importance à la qualité du risque-client.}

Malheureusement, cette seconde exigence est contradictoire avec une relation globale dans la mesure où le risque-client, considéré comme trop grand doit être partagé avec d'autres. Aussi refait surface l'idée de recréer des regroupements financiers formalisés dans lesquels le partenariat se couple avec le partage du risque. Les banques comme les entreprises n'ont guère d'autres possibilités dans la période actuelle.

Des évolutions identiques se sont produites en dehors du cercle des banques dans le domaine des capitaux permanents des entreprises. Qu'il s'agisse du second marché où, à côté de trop rares réussites, sont intervenues de nombreuses disparitions d'entreprises de moyenne dimension considérées comme prometteuses, ou encore des sociétés de capital de risque qui, malgré une sélection rigoureuse des dossiers, ont connu de multiples déboires. Par ailleurs, le décompte des LBO ou RES encore en vie montre à l'évidence que l'effet de levier, idéal sur le papier, est d'une fragilité extrême en cas de dégradation de la conjoncture. Pour terminer, nous citerons les Sociétés de développement régional, outil idéal d'accompagnement des entreprises sur le plan local soit en fonds propres, soit en capitaux permanents. Leur parcours, pour le moins heurté au cours des années récentes, montre bien que, malgré leur proximité dans l'analyse des dossiers, la mesure du risque et son appréciation sur la durée sont difficiles. 


\subsection{Des mesures pour favoriser l'accès des PMI aux financements}

Si l'analyse statistique fait ressortir des situations grandes entreprises/PMI relativement comparables, le constat pour les années récentes montre dans la réalité un niveau de risque sensiblement supérieur. Cette asymétrie nous semble tenir à de multiples éléments :

- meilleure appréhension des marchés et gammes de produits plus étoffées dans les grandes entreprises permettant des adaptations plus rapides aux évolutions des marchés ou de la conjoncture ;

- pour les grandes entreprises, un accès plus large aux divers compartiments des marchés financiers et une "solidarité », parfois contrainte, des financiers ; l'exemple d'EuroDisney en est un témoignage récent ;

- une discipline dans la gestion prévisionnelle et la communication financière qui avantage considérablement les grandes entreprises; les cas d'entreprises moyennes ayant failli à leurs obligations en matière d'informations financières sont nombreux et, en règle générale, ont conduit à une coupure complète avec le marché financier. En outre, de trop nombreux dirigeants de PMI répugnent à ouvrir leurs livres, à faire part de leurs projets ou à partager le pouvoir dans l'entreprise ;

- un effet «volume» de financements ou de capitaux demandés qui pourrait être favorable aux PMI (plusieurs petits dossiers partagent les risques), mais qui en définitive se retourne contre elles : nécessité d'analyser et de suivre plusieurs dossiers, risque de défaillance individuelle plus élevé, etc.

À cette asymétrie dans la demande de capitaux répond une asymétrie dans l'offre. À l'évidence, les montants élevés de capitaux disponibles pour les grands projets ne sont pas présents pour répondre aux besoins des PMI. Dans le schéma anglo-saxon, la réponse est en partie assurée par la mobilisation de l'épargne de proximité. Ce maillon manque dans le dispositif français. Les SDR ont montré leurs limites et il n'est pas certain qu'elles renaissent de leurs cendres. Les sociétés de capital de risque privées ou filiales de banques sont trop souvent éloignées de la grande masse des PMI.

Pour ce qui concerne les banques, les financements doivent s'inscrire dans le cadre réglementaire qui s'impose à elles, à savoir que les banques ne doivent pas mettre en jeu les dépôts de leurs clients. Le risque qu'elles peuvent prendre ne peut donc être que mesuré. Au-delà d'un niveau raisonnable de fonds propres que le marché doit pouvoir fournir, la restauration d'un véritable partenariat basé sur la confiance entre banques et entreprises et le partage des 
risques dans le cadre d'un regroupement financier sont, dans le contexte actuel, la voie la plus sûre pour que les entreprises disposent des financements qui leur sont nécessaires.

\section{Conclusion}

En définitive, outre la nécessité impérative de s'assurer d'une définition cohérente et précise de l'autonomie financière, il apparaît qu'une fois un certain montant de fonds propres acquis, l'augmentation de celui-ci n'est la garantie ni d'un plus grand dynamisme en matière d'investissement ni d'une gestion plus favorable à l'emploi ${ }^{25}$.

Avec les grandes firmes, le risque pris par les apporteurs de capitaux porte plus sur la masse de capitaux mobilisés que sur la valeur intrinsèque du projet, surtout que le prêteur bénéficie d'une information d'autant plus riche que l'entreprise est de grande taille.

Les prêteurs (voire les apporteurs de capitaux en général) considèrent les PMI comme plus risquées que les grandes entreprises. Cette appréciation, trop souvent réduite au seul constat d'une insuffisance de fonds propres, se justifie essentiellement par des prises de risques par les PMI effectivement plus fortes, comme le montre leur effort de renouvellement du potentiel productif plus important que dans les grandes entreprises.

Toutefois, la meilleure rentabilité des capitaux offerte par les PMI devrait constituer en elle-même une garantie pour les prêteurs. Aussi, l'écart de taux d'intérêt observé selon la taille des entreprises ne peut se justifier par des différences a priori de performances économiques et financières, mais bien par un taux de risque supérieur.

Pour finir, les PMI ne seraient-elles pas handicapées par la crédibilité de l'information délivrée concernant leurs projets et leur capacité à prouver leur pérennité ? Cette faiblesse relèverait autant de leur responsabilité que de celle de leurs partenaires financiers. Elle ne pourrait être palliée que par un système de financement et une gestion adaptés à leurs spécificités tenant compte du fait que leur principale contrainte est celle des débouchés qui conditionnent le retour sur investissement.

25. Voir les résultats des différentes études présentées à l'occasion de la $18^{\mathrm{e}}$ Journée des Centrales de Bilans, Paris, novembre 1993. 


\section{Bibliographie}

ARTus, P. (1992), «Politique salariale et taux d'intérêt en France dans les années 80: peut-on envisager de poursuivre sur la lancée?», Revue Économique, p. 450-486.

BARDos, M. et J. CORDIER (1991), «Éléments d'analyse de la situation financière des entreprises», Fiche thématique no 1, Conseil National du Crédit, p. 295-331.

DE BANDT, O. et P. JACQUINOT (1991), «Le mode de financement des entreprises », Bulletin trimestriel de la Banque de France, $3^{c}$ trimestre, p. 191-199.

FAyolle, J. et H. PelerauX (1994), «Entreprises: une situation à double face», Lettre de l'OFCE, $\mathrm{n}^{\circ} 114,21$ avril.

ICARD, A. (1991), «L'épargne à long terme», Bulletin trimestriel de la Banque de France, $4^{\mathrm{e}}$ trimestre, p. 107-122 bis.

MANCEAU, B. (1992), Analyse financière normative des entreprises, Paris, La Revue Banque Éditeur, p. 120-121.

Modigliani, F. et M.H. MiLler (1958), «The cost of capital, corporate finance and the theory of investment », American Economic Review, mai $n^{\circ} 8$.

PARANQUE, B. (1992), Contraintes économiques des PMI et des grandes entreprises, 1981-1990, Centrale de Bilans, Paris, Banque de France, p. 30.

PARANQUE, B. (1994a), Emploi, accumulation et rentabilité financière, Paris, Banque de France, p. 7.

PARANQUe, B. (1994b), «Fonds propres, rentabilité et efficacité chez les PMI. Méthodes d'analyse et appréciation des situations financières ", Revue d'Économie Industrielle, $\mathrm{n}^{\circ}$ 67, $1^{\text {er }}$ trimestre, p. 175-190.

ViEILlE, J.N. (1992), «Mode de croissance et financement des principaux groupes français (1983-1990)», Analyse financière, p. 67-75. 


\section{Lexique}

Apports externes

Endettement

+ Groupe et associés dans dettes financières

Capacité d'autofinancement

Excédent brut global

- Intérêts sur endettement, y compris intérêts dans loyers de crédit-bail

- Intérêts sur groupes et associés

- Impôts sur les bénéfices

Capital engagé

Financement propre,

(y compris immobilisations en crédit-bail amorties)

+ Groupe et associés

+ Endettement, y compris crédit-bail

Crédits bancaires courants (y compris intérêts courus)

Découverts

+ Mobilisations de créances

+ Effets escomptés non échus

+ Affacturage

+ Crédits de trésorerie

\section{Emprunts}

Emprunts bancaires (y compris immobilisations en crédit-bail non amorties)

+ Emprunts participatifs

+ Emprunts obligataires + titres participatifs

+ Autres emprunts (État, FDES, fonds de participation, etc.)

\section{Endettement}

Emprunts

+ Crédits bancaires courants

Excédent Brut Global (EBG)

Excédent brut d'exploitation

+ Produits et charges hors exploitation

Financement propre

Capital et primes

+ Réserves

+ Résultats

+ Subventions d'investissement

+ Amortissements, y compris immobilisations en crédit-bail amorties

Réserves

d'autofinancement

+ Provisions pour dépréciation d'actif

+ Provisions réglementées

+ Provisions pour risques et charges, sauf congés payés 
Investissements productifs

Investissements en immobilisations corporelles d'exploitation

+ Immobilisations nouvelles en crédit-bail

Réserves d'autofinancement

Réserves, résultat, subventions d'investissement

+ Amortissements

+ Provisions

Valeur ajoutée $C D B(V A C D B)$

Chiffres d'affaires

+ Production stockée

+ Production immobilisée

+ Subvention d'exploitation

+ Variation des stocks d'approvisionnements et de marchandises

- Achats d'approvisionnements et marchandises

- Autres achats et services extérieurs

+ Annuités de crédit-bail

+ Dépenses de personnel extérieur

- Impôts et taxes

Valeur ajoutée globale (VAG)

Valeur ajoutée CDB

+ Produits et charges hors exploitation 